\title{
Efficient MRI Segmentation and Detection of Brain Tumor using Convolutional Neural Network
}

\author{
Alpana Jijja ${ }^{1}$, Dr. Dinesh Rai ${ }^{2}$ \\ Computer Science and Engineering, Ansal University, \\ Gurugram, Haryana, India.
}

\begin{abstract}
Brain tumor is one of the most life-threatening diseases at its advance stages. Hence, detection at early stages is very crucial in treatment for improvement of the life expectancy of the patients. magnetic resonance imaging (MRI) is being used extensively nowadays for detection of brain tumors that requires segmenting huge volumes of 3D MRI images which is very challenging if done manually. Thus, automatic segmentation of the images will significantly lessen the burden and also improve the process of diagnosing the tumors. This paper presents an efficient method based on convolutional neural networks (CNN) for the automatic segmentation and detection of a brain tumor using MRI images. Water cycle algorithm is applied to CNN to obtain an optimal solution. The developed technique has an accuracy of $98.5 \%$.
\end{abstract}

Keywords-Brain tumor; segmentation; convolutional neural network; water cycle algorithm

\section{INTRODUCTION}

Brain, the most important part has the most complex structure in the body. The presence of the skull around the brain hinders the study of its functions and also increases the complexity of diagnosing the diseases [1]. The brain is not prone to any particular diseases like the other parts of the body but can be triggered by the abnormal growth of cells in which there is a change in its behavior and structure. This abnormality is usually an indication of Brain Tumor. MRI is used for the detection of such tumors. With the improvements in technology, the segmentation of brain tumor and tissue has become an actively researched area [2]. The major issue in the segmentation of images is the clustering of feature vectors which are similar. Thus, extraction of acceptable features is the primary requirement for successfully segmenting the images. The useful feature extraction of images is a difficult task due to the intricacies in the structures of the various tissues in the brain [3]. The image segmentation is a significant building block in the studies related to brain tumor because, the extent of segmented brain tumor can remove the confusing structures from other brain tissues thus providing higher accuracy in the classification of the subtypes of the tumor and give information about the diagnosis and can also effectively monitor the growth, recurrence or shrinking of the tumors. The techniques of image segmentation can be classified as based on region/surface growing, edge detection, classifiers, quantization or feature clustering of vectors. The technique of vector quantization is an efficient model for the process of segmenting [4]. This technique will partition the ndimensional vector space to $M$ regions for optimizing the criterion function. The vector quantization involves two processes of training and encoding, where training will determine the codebook vector set based on the input data probability while encoding will assign the input vectors to the vectors present in the codebook.

\section{RELATED WORK}

Segmentation of images in the applications such as detection of abnormality, surgical planning, post-surgery assessment in the medical field is a significant task. Hence, many methods have been proposed for segmentation [2] revisited the efficacy of two distinct texture features along with the application of the multimodal MR images for segmenting and classifying the pediatric brain tumors. The fractal feature of one of the texture is derived using PiecewiseTriangular-Prism-Surface-Area (PTPSA) algorithm and the other texture extraction uses fractional Brownian motion algorithm. The fusion of the features is achieved through a self-organizing map (SOM). The experimental results suggested that fusing the features of the intensity and fractal wavelet of the multimodal MR images gave better results when compared to that in images with a single modality. Mustaqeem et al., [5] developed and introduced an effective algorithm for detecting the brain tumor. The developed segmentation method was based on thresholding and watershed techniques. The images of the human brains obtained from the MRI scans were used for the segmentation process but this algorithm cannot be used in the segmentation of 3D images.

Padole \& Chaudhari [6] developed a method for detecting the brain tumors from MRI images through component analysis. This method which can automatically detect the brain tumor surface area is a combination of Normalized cut (Ncut) and mean shift algorithms. Roy \& Bandyopadhyay detected and measured the tumor from the brain MRI using symmetric analysis [7]. The very application of quantitative analysis of this method helps in the identification of disease status. El-Dahshan et al., reviewed the various classification and segmentation techniques and inferred that computer-aided diagnosis is the major issue of the MRI of the human brain [8]. Hence, the authors developed a hybrid intelligent machine learning method for automatically identifying the tumor through MRI brain. The images are segmented using a neural network which is coupled with a feedback pulse. The classification of the images to abnormal and normal is achieved with the help of a feed-forward neural network with backpropagation. The experiments are conducted on 101 images comprising 87 abnormal and 14 normal images from 
the MRI dataset of a human brain. The results showed that this technique gave an accuracy of $99 \%$.

Abdel-Maksoud et al., introduced an image segmentation technique that used the integration of K-means clustering and fuzzy C-means algorithm [9]. The stages of thresholding and segmentation at a set level helped in detecting the tumor accurately. The computational time was lower due to use of $\mathrm{K}$-means clustering and accuracy was higher because of the use of fuzzy c- means. The performance evaluation of the suggested technique was processed by comparing it to the existing methodologies with respect to performance, processing time and accuracy. The results from the experiments proved the method's effectiveness in dealing with more number of problems related to segmentation by the improvement in the quality of segmentation and accuracy with minimum run time. Menze et al., [10] reported the outcomes of the Multimodal Brain Tumor Image Segmentation Benchmark (BRATS). A set of 65 multi-contrast MRI scans of various glioma patients was used as a dataset for around twenty segmentation algorithms for detecting the tumors. The authors found that separate algorithms were suitable for differentiated regions of the tumor and no particular algorithm was found best for all the subregions. Mahalakshmi \& Velmurugan [11] developed an algorithm to detect brain tumor using particle swarm optimization. The algorithm comprises of four stages the conversion, implementation, selection, and extraction. The proposed PSO algorithm helps in determining the size of the MRI images of the brain. Elamri \& Planque proposed a new algorithm for segmenting the Glioblastoma (GBM) tumor based on the 3D convolutional neural networks [12]. The authors proposed the generalization of CNN's to obtain 3D filters for increasing the robustness and conserving the spatial information. The presented CNN architecture also enabled the expansion of the effective size of data and reduction in the variance of the developed model and its accuracy was found to be $89 \%$. Pereira et al., introduced an automatic technique for segmentation using convolutional neural networks (CNN) which explored small $3 * 3$ kernels [13]. The use of these kernels allowed the deeper design of the architecture and had a positive impact against overfitting. By utilizing the intensity normalization as a preprocessing step along with augmentation of the data demonstrated the effectiveness of the segmentation for MRI images. This technique is validated using the BRATS 2013 database. The main problem of improvement in accuracy, still pertains in the above mentioned work. This can be solved by using optimized technique using WCA.

A completely automatic technique for segmenting the brain tumor which used deep convolutional networks based on U-Net was developed by Dong et al., [14]. The experiments were carried out on BRATS 2015 datasets consisting of both LGG as well as HGG patients. The results obtained from the experiments demonstrated that the proposed technique was effective. The technique was validated using the scheme of five-fold cross-validation. Using this technique, a model for segmenting the tumor images for specific patients can be generated without manually interfering. Havaei et al., [15] put forward an automatic technique for segmentation of brain tumor using high capacity, flexible deep neural networks (DNN). This developed DNN technique uses both the global as well as the local contextual features at the same time. The difficulties with respect to the imbalance in the labels of the tumor are eliminated using a training procedure comprising two phases.

\section{RESEARCH METHODOLOGY}

The flow diagram for the proposed Brain Tumor image segmentation using Optimal Convolutional Neural Network (OCNN) is shown in Fig. 1. This proposed technique is implemented using MATLAB 14a software. The database contains grayscale images of the human brain. The images are preprocessed from the database using the technique of median filtering to remove the noise and reduce the distortions. The next step is the extraction of patches. A corner, edge or the uniform texture of the image can be considered as the patch. The extraction of patches helps in determining the parts of the brain where the abnormality is present. Further in patch preprocessing the values of standard deviation, variance, intensity etc. of the images which are under the training phase are obtained. The filtered images in which the tumors are present are detected, classified, and clustered using the CNN. Images were clustered using the K-means clustering technique and segmented using morphological segmentation. Water cycle optimization algorithm is applied to calculate the weight and bias factor that has a significant role in identifying and classifying of the tumors. The factors of weight and bias help in classifying the images with higher accuracy. Lastly, performance of this method is evaluated for different factors like specificity, accuracy, sensitivity, entropy, etc.

\section{A. Image Preprocessing}

Preprocessing enhances the quality of the images by eliminating noise and reducing the distortions of the input image. The current study uses a median filtering technique to preprocess the image. A median filter is a non-linear filter based on statistics. Subsequently the noisy value of the image is replaced by the median value of the neighboring mask. The pixels of the mask are ranked in the order of Grey levels, and the median value of the group is stored to replace the noisy value. The output of the median filtering is:

$$
g(x, y)=\operatorname{med}\{f(x-i, y-j), i, j \in W\}
$$

Where:

$$
f(x, y)=\text { original image and } g(x, y)=\text { output image }
$$

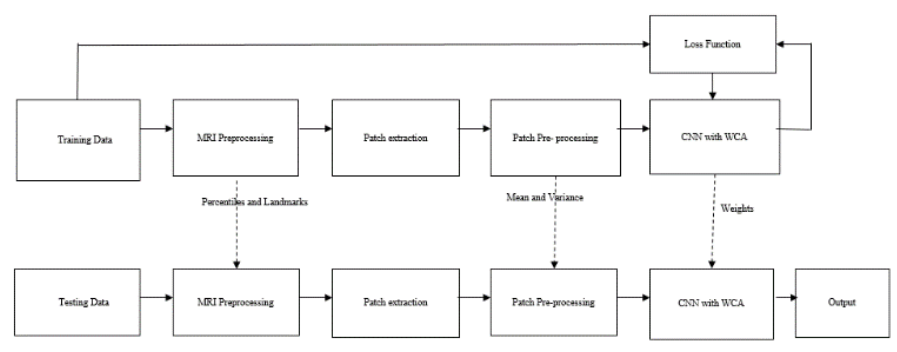

Fig. 1. Proposed Workflow Diagram. 
$\mathrm{W}=2 \mathrm{D}$ mask whose shape can be square, circular, linear etc. The median filter will move throughout the image, pixel by pixel and replaces each value with the median value of the neighboring pixels. This matrix of neighbors is referred to as "windows". The window will slide over the complete image pixel by pixel. The median value is derived by sorting all the values of the pixel from the window in the numerical order and then transposing the pixel under consideration to the median pixel value.

\section{B. Convolutional Neural Networks (CNN)}

Fig. 2 illustrates that $\mathrm{CNN}$ which is feed forward neural network and is widely used for image recognition. This is made up of neuron with learnable weights and biases. Each neuron receives several inputs, takes a weighed sum over them, pass it through activation function and responds with the output. CNN operates over volumes like neural networks where the input is a vector but in case of CNN the input is a multi-channeled image. $\mathrm{CNN}$ has overcome image segmentation challenges by automatically learning a hierarchy of increasingly complex features directly from data. The CNN basically is used in convolving the image with the kernels for the process of obtaining the feature maps. The weights in the kernels help in connecting each unit of the feature map to the previous layers. These weights of the kernels are utilized during training of the datasets for improving the attributes of the input [16]. The number of weights which have to be trained in the convolutional layers is lesser when compared to the fully connected (FC) layers because the kernels are common to all the units of one particular feature map. Some of the significant concepts with respect to CNN are discussed below.

- Initialization is the most significant step for accomplishing convergence. This process helps in maintaining the gradients at the required levels else there will be a chance of explosion of the gradients that are back propagated.

- The activation function is accountable for the data transformation in a nonlinear manner. There are various types of activation function of which the modified Rectifier linear units (ReLU) is used. It is defined as

$$
f(x)=\max (0, x)+\alpha \min (0, x)
$$
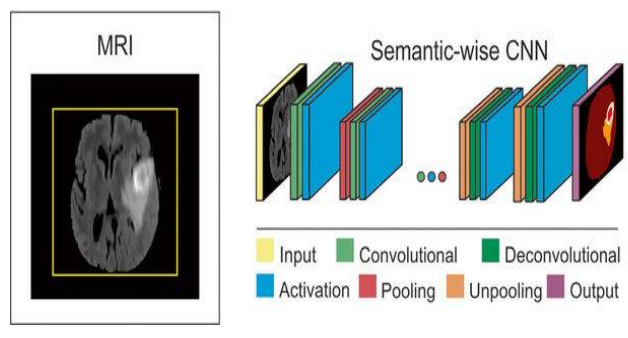

Fig. 2.
- In the feature maps, the process of pooling combines the feature that is nearby spatially. This sequence of redundant features helps in making the representation invariant in the case of small changes and also more compact. The computation load for the successive stages is also reduced.

- The overfitting is decreased with the help of regularization. In every step of training, it will eliminate the nodes of the network. In this way, all the nodes in the Fully Connected layer are forced to learn better representations and also prevent the coadaptation of nodes.

- The number of parameters to train a fully connected network is much larger as compared to a CNN. In most images the nearby pixels are generally related but a fully connected neural network doesn't take this into account while a CNN takes into account relationship between space and pixel within an image.

- The loss function is the function $(\mathrm{H})$ that has to be reduced during the training phase of the data. Categorical Cross-entropy is used in this study [17].

$$
\mathrm{H}=-\sum_{j \in \text { voxels }} \sum_{k \epsilon \text { voxels }} c_{j} \log \left(\widehat{c_{J, k}}\right)
$$

The WCA off-late has been developed as the optimization technique which can be applied to various engineering design constrained optimization problems [18]. The fundamental concept which has been used in the development of this algorithm is based on the actual water cycle process. The WCA plays an important role in Identifying, detecting and classifying the images with tumors. The pseudocode for the WCA is given below [19].

\section{RESUlTS AND Discussions}

The input to the proposed technique is the greyscale images obtained from the database related to brain tumor obtained from the internet. The images from the database are split into two sets of training and testing images. Totally 400 images of the brain MRI is used, out of which 300 images are considered for training and 100 images for testing. Fig. 3 shows the greyscale image of the human brain which is considered as an input obtained from the testing set of images. This image is preprocessed using the technique of median filtering to remove the noise and reduce the distortions present in the input image. Fig. 4 is filtered image of the test image.

The next step is the patch processing. A corner, edge or the uniform texture of the image can be considered as the patch. The size of each patch is 10px by 10px. Fig. 5 shows the patch processed image. This process is carried out to find the parts that have abnormalities. Further, the patch pre-processing is carried out to obtain the values of standard deviation, variance, intensity etc. of the images which are under the training phase.

Architecture of CNN. 


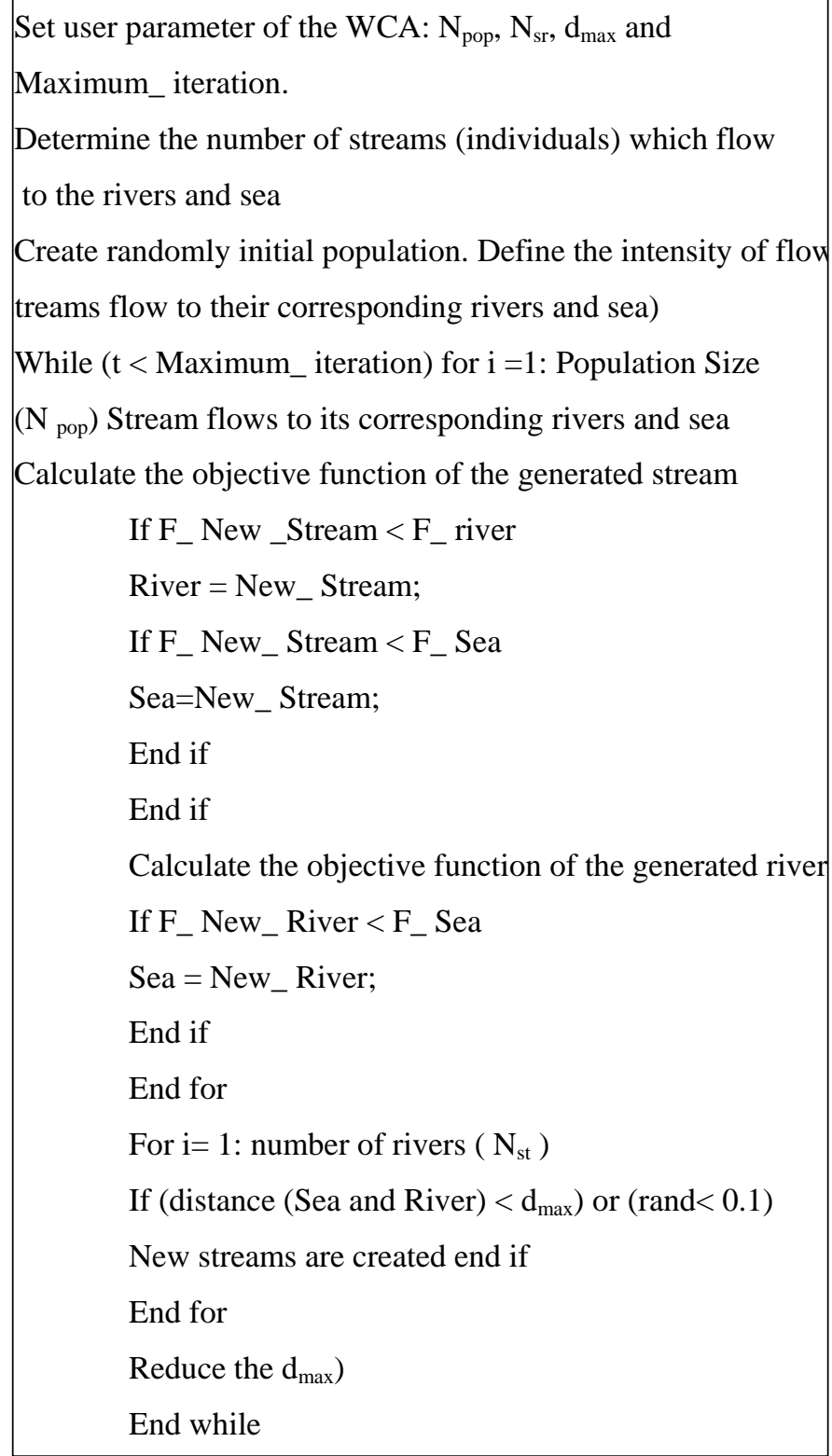

Fig. 6 shows the patch preprocessed image. The final step is the cluster based segmentation of the tumor images using $\mathrm{CNN}$. The CNN will classify the images based on various factors like intensity, shape etc. and detect the tumor. Fig. 7 is the clustered images to the brain showing various edges.

The brain tumors are usually formed by large connected regions. The final step of post-processing includes segmenting, detecting and extracting the tumor from the image and will remove any of the connected components however small the components are. At this stage, the tumor is detected along with the area that is covered by the disease. Fig. 8 shows the final output of CNN. Fig. 8a shows only the tumor part of the image and the Fig. $8 \mathrm{~b}$ shows the color based segmented image.

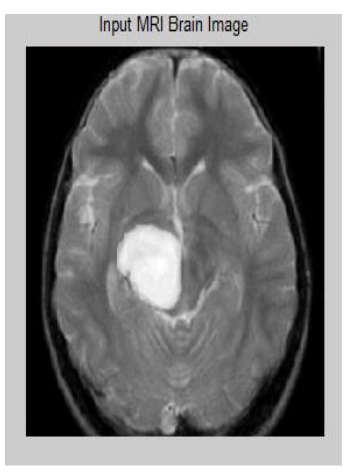

Fig. 3. Input image.

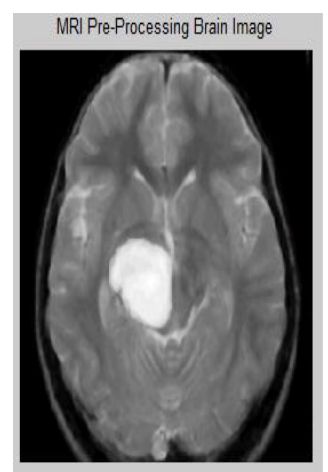

Fig. 4. Median Filter Image.

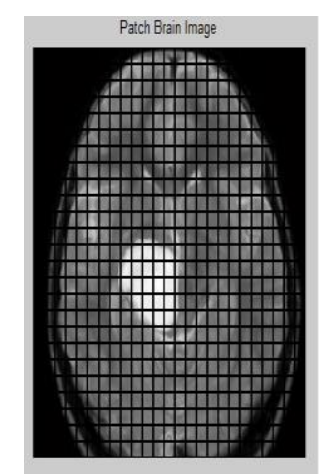

Fig. 5. Patch Image.

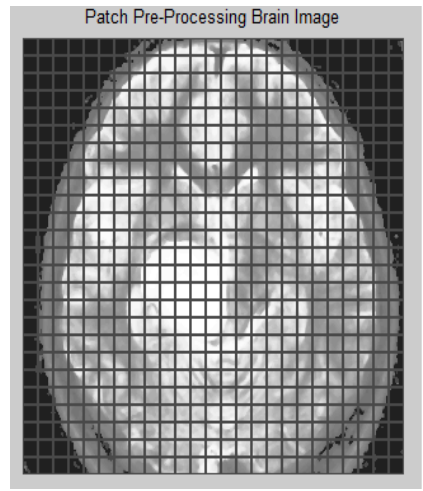

Fig. 6. Patch Pre-Processing Image. 


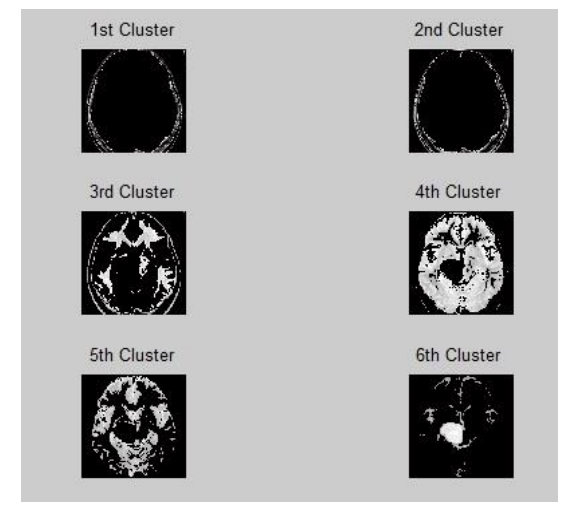

Fig. 7. Showing the Detection of the Tumor by Clustering.

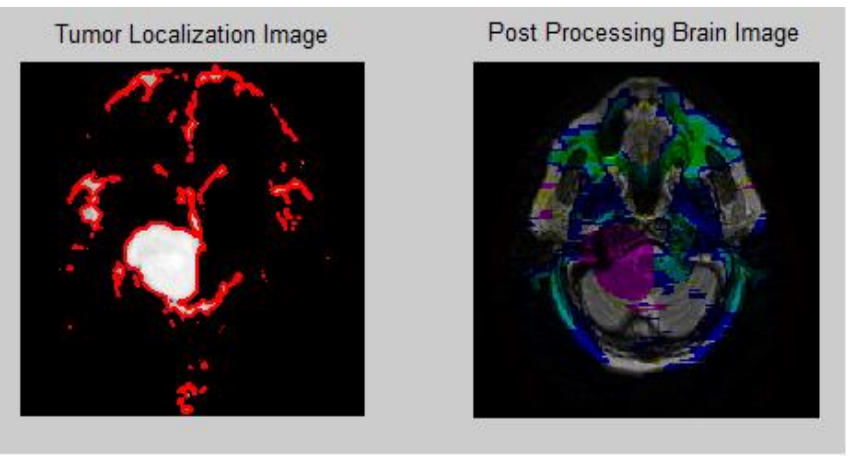

Fig. 8. (a) Localized Tumor Image (b) Post Processed Brain Image.

\section{A. Performance Evaluation}

The performance of the proposed technique is evaluated in terms of accuracy, sensitivity, and specificity where:

TP $=$ True Positive

$\mathrm{FN}=$ False negative

The sensitivity is defined as the ability to determine the tumors correctly. It can be calculated using the following relation.

\section{Sensitivity $=\mathrm{TP} /(\mathrm{TP}+\mathrm{FN})$}

The Specificity is defined as the ability for the correct determination of healthy cases. It can be calculated using the following relation.

\section{Specificity $=\mathrm{TN} /(\mathrm{TN}+\mathrm{FP})$}

Accuracy is defined as the ability to differentiate healthy and unhealthy cases correctly can be calculated using the following relation.

\section{Accuracy $=(\mathrm{TN}+\mathrm{TP}) /(\mathrm{TN}+\mathrm{TP}+\mathrm{FN}+\mathrm{FP})$}

The performance metrics calculated for the tested images is tabulated in Table I. and Fig. 9 shows the plot for the sensitivity, specificity and accuracy parameters.

The comparative study in Table II examines the various automated segmentation techniques used by researchers on brain MRI. It has been observed that the proposed WCA algorithm has performed and overcome image segmentation challenges by automatically learning a hierarchy of increasingly complex features directly from the data. In this algorithm is shown the calculation of cost sensitive feature selection using loss equation which balances accuracy and computational cost. Hence more modern techniques can be used be increase the accuracy be using deep learning methods along with increased sample data size.

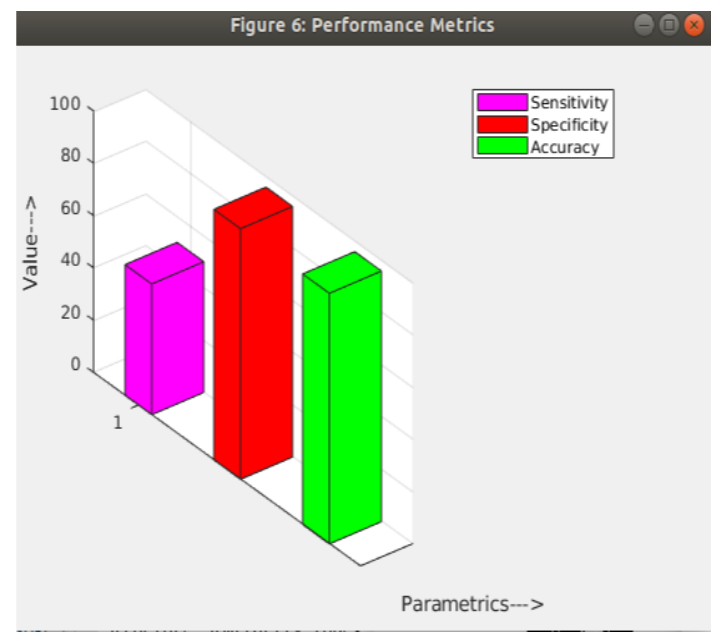

Fig. 9. Plot of Performance Metrics.

TABLE I. PERFormance MATRIX

\begin{tabular}{|l|l|}
\hline Parameter & Value \\
\hline Tumor segment area & 18.512 \\
\hline Tumor positive value & 1 \\
\hline True negative value & 60220 \\
\hline False positive value & 886 \\
\hline Sensitivity & 50 \\
\hline Specificity & 98.55 \\
\hline Accuracy & 98.54 \\
\hline MSE & 9.82 \\
\hline PSNR & 38.20 \\
\hline Entropy & 0.771 \\
\hline Correlation & 0.194 \\
\hline Structure similarity index & 0.2108 \\
\hline
\end{tabular}

TABLE II. COMPARATIVE SUMMARY OF DIFFERENT CLASSIFIER FOR SEGMENTATION ACCURACY

\begin{tabular}{|l|l|l|}
\hline Title & Technique & Accuracy \\
\hline $\begin{array}{l}\text { Tumor segmentation from single } \\
\text { contrast MR images of the human } \\
\text { brain [20] }\end{array}$ & $\begin{array}{l}\text { Random forest } \\
\text { classification }\end{array}$ & $83.00 \%$ \\
\hline $\begin{array}{l}\text { Detection of Brain Tumor in MRI } \\
\text { Images, using Combination of Fuzzy } \\
\text { C-Means and SVM [21] }\end{array}$ & $\begin{array}{l}\text { Fuzzy c-means } \\
\text { algorithm and SVM } \\
\text { classifier }\end{array}$ & $91.66 \%$ \\
\hline $\begin{array}{l}\text { An Automatic Brain Tumor } \\
\text { Extraction System Using Different } \\
\text { Segmentation Methods [22] }\end{array}$ & $\begin{array}{l}\text { Fuzzy c-means } \\
\text { algorithm }\end{array}$ & $90.57 \%$ \\
\hline $\begin{array}{l}\text { Tumor detection and classification } \\
\text { of MRI brain image using wavelet } \\
\text { transform and SVM [23] }\end{array}$ & $\begin{array}{l}\text { Wavelet transform } \\
\text { and SVM classifier }\end{array}$ & $86 \%$ \\
\hline Proposed technique & $\begin{array}{l}\text { CNN with WCA } \\
\text { algorithm }\end{array}$ & $98.50 \%$ \\
\hline
\end{tabular}




\section{CONCLUSION}

The current study developed an automated method for the segmentation and detection of a brain tumor. The greyscale images obtained from the database are preprocessed using median filtering to remove the noise and distortions that are present in the input image. The $\mathrm{CNN}$ is then used for clustering and segmenting the images and finally to detect the tumor. The WCA optimization algorithm is applied for optimal clustering of the images. The developed automatic image segmentation and tumor detection technique are found to have an accuracy of $98.5 \%$, sensitivity of $50 \%$ and specificity of $98.5 \%$. The comparison of the proposed technique with existing methods proves that the technique developed in this paper has the highest accuracy. The accuracy can be further improved using large datasets, however as compared to their imaging area, availability of dataset is not easily available. Thus sharing the data resources by different healthcare service providers may help to overcome this issue. There are unlimited opportunities to improve healthcare system by using more sophisticated filters in CNN. While the proposed model yields promising results, and leaves no room for errors. In surgical setting it is essential to remove as much of tumor mass as possible without damaging the surrounding healthy tissue. In future more work could be extended by developing more advanced patch extraction algorithm which will help in segmentation accuracy.

\section{REFERENCES}

[1] Gondal and M. Khan, "A review of fully automated techniques for brain tumor detection from MR Images", International Journal of Modern Education and Computer Science, vol. 5, no. 2, pp. 55-61, 2013.

[2] K. Iftekharuddin, K.M, "Techniques in fractal analysis and their applications in brain MRI", Medical Imaging Systems Technology: Volume 1: Analysis and Computational Methods,2005, pp. 63-86.

[3] Wang and D. Doddrell, "A segmentation-based and partial-volumecompensated method for an accurate measurement of lateral ventricular volumes on T 1 -weighted magnetic resonance images", Magnetic Resonance Imaging, vol. 19, no. 2, pp. 267-273, 2001.

[4] Krishnamurthy, S. Ahalt, D. Melton and P. Chen, "Neural networks for vector quantization of speech and images", IEEE Journal on Selected Areas in Communications, vol. 8, no. 8, pp. 1449-1457, 1990.

[5] Mustaqeem, A. Javed and T. Fatima, "An efficient brain tumor detection algorithm using watershed \& thresholding based segmentation", International Journal of Image, Graphics and Signal Processing, vol. 4, no. 10, pp. 34-39, 2012.

[6] V. Padole and D.S. Chaudhari, "Detection of the brain tumor in MRI images using the mean shift algorithm and normalized cut method", International journal of Engineering and Advanced Technology, pp. 52-56, 2012.

[7] S. Roy and S. Bandyopadhyay, "Detection and quantification of brain tumor from MRI of brain and it's symmetric analysis", International Journal of Information and Communication Technology Research, vol. 2, no. 6, pp. 477-483, 2012. [Accessed 30 March 2019].
[8] E. El-Dahshan, H. Mohsen, K. Revett and M. Salem, "Computer-aided diagnosis of human brain tumor through MRI: A survey and a new algorithm", Expert Systems with Applications, vol. 41, no. 11, pp. 55265545, 2014. Available: 10.1016/j.eswa.2014.01.021.

[9] Abdel-Maksoud, M. Elmogy and R. Al-Awadi, "Brain tumor segmentation based on a hybrid clustering technique", Egyptian Informatics Journal, vol. 16, no. 1, pp. 71-81, 2015.

[10] Menze et al., "The multimodal brain tumorimage segmentation benchmark (BRATS)", IEEE transactions on medical imaging, vol. 34, no. 10 , pp. 71-81, 1993.

[11] S. Mahalakshmi and T. Velmurugan, "Detection of brain tumor by particle swarm optimization using image segmentation", Indian Journal of Science and Technology, vol. 8, no. 22, 2015.

[12] Elamri, and T.de Planque, "A new algorithm for fully automatic brain tumor segmentation with 3-D convolutional neural networks", 2016.

[13] S. Pereira, A. Pinto, V. Alves, and C. A. Silva, "Brain tumor segmentation using convolutional neural networks in MRI Images," IEEE Transactions on Medical Imaging, vol. 35, no. 5, pp. 1240-1251, 2016.

[14] H. Dong, G. Yang, F. Liu, Y. Mo, and Y. Guo, “Automatic brain tumor detection and segmentation using U-Net based fully convolutional networks," Communications in Computer and Information Science Medical Image Understanding and Analysis, pp. 506-517, 2017.

[15] M. Havaei, A. Davy, D. Warde-Farley, A. Biard, A. Courville, Y. H. Tang, H. Lu, W. Liu, and X. Tao, "Tumor segmentation from single contrast MR images of human brain," IEEE 12th International Symposium on Biomedical Imaging (ISBI), 2015.

[16] K. O'Shea and R. Nash, "An introduction to convolutional neural networks “- arXiv preprint arXiv:1511.08458., 2015.

[17] S. Cui, L. Mao and S. Xiong, "Brain tumor automatic segmentation using fully convolutional Networks", Journal of Medical Imaging and Health Informatics, vol. 7, no. 7, pp. 1641-1647, 2017.

[18] H. Eskandar, A. Sadollah, A. Bahreininejad, and M. Hamdi, "Water cycle algorithm - A novel metaheuristic optimization method for solving constrained engineering optimization problems," Computers \& Structures, vol. 110-111, pp. 151-166, 2012.

[19] Sadollah, H. Eskandar, and J. H. Kim, "Water cycle algorithm for solving constrained multi-objective optimization problems," Applied Soft Computing, vol. 27, pp. 279-298, 2015.

[20] M. Havaei, A. Davy, D. Warde-Farley, A. Biard, A. Courville, Y. H. Tang, H. Lu, W. Liu, and X. Tao, "Tumor segmentation from single contrast MR images of human brain," IEEE 12th International Symposium on Biomedical Imaging (ISBI), 2015.

[21] Parveen and A. Singh, "Detection of brain tumor in MRI images, using combination of fuzzy c-means and SVM," 2nd International Conference on Signal Processing and Integrated Networks (SPIN), 2015.

[22] A. Kaur, "An automatic brain tumor extraction system using different segmentation methods," Second International Conference on Computational Intelligence \& Communication Technology (CICT), 2016.

[23] R. Mathew asssnd P. B. Anto, "Tumor detection and classification of MRI brain image using wavelet transform and SVM," International 